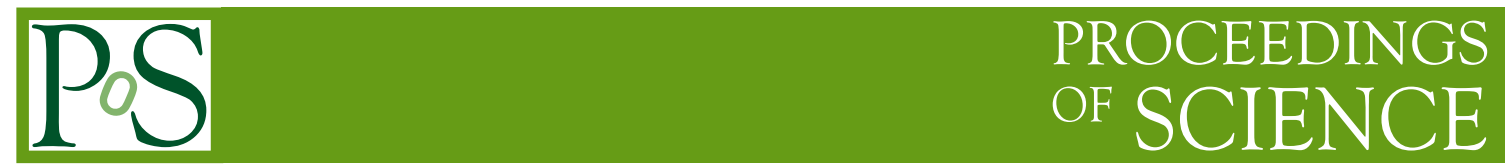

\title{
Hypernuclear physics at storage rings
}

\author{
A. Feliciello* \\ Istituto Nazionale di Fisica Nucleare, Sezione di Torino \\ Via P. Giuria 1, I-10125 Torino, Italy \\ E-mail: Alessandro.Feliciello@to.infn.it
}

Traditionally hypernuclear physics experiments are fixed target ones and they are carried out at hadron accelerators with extracted pion and/or kaon beams. However storage rings sometimes offer peculiar, or even unique, experimental conditions that represent essential requirements for the success of the proposed scientific program. Then such machines are an interesting, alternative playground where to perform hypernuclear measurements following a non conventional experimental approach.

8th International Conference on Nuclear Physics at Storage Rings-Stori11,

October 9-14, 2011

Laboratori Nazionali di Frascati dell'INFN, Italy

\footnotetext{
* Speaker.
} 


\section{Introduction}

Hypernuclear physics (or strangeness nuclear physics) studies bound nuclear systems, known as hypernuclei [1], where one of the constituent nucleons is replaced by one (or two) hyperon(s), typically $\Lambda$ particle(s). On the one hand high resolution spectroscopy of such systems basically represents the unique method to infer information about the hyperon-nucleon interaction at low energies [2]; on the other the observation of their decay modes offers the only way to investigate the four-baryons, strangeness changing, $\Lambda \mathscr{N} \rightarrow \mathscr{N} \mathscr{N}$ weak vertex [3]; vivid interest has been then exited by the possible existence of (deeply) bound kaonic state $K^{-} p p$ [4]; another interesting topic that could be addressed is that of the neutron-rich nuclei, produced thanks to the "glue role" of the $\Lambda$ particle [5]; finally, besides considering the hyperon like a probe exploring the nuclear structure, it is possible as well to look at the other side of the coin by studying how the nuclear medium could affect the properties of the embedded hyperon.

\section{Consolidated activities}

\subsection{The COSY-13 experiment}

One of the first observables on which experimentalists focussed their attention is the hypernucleus lifetime $\tau$ which is intimately connected to the measurement of total decay width of the system. Among all the measurable quantities $\tau$ is the one that can be measured with the highest accuracy. It has been found that the lifetime of a $\Lambda$ hyperon bound in a hypernucleus is clearly affected by the nuclear medium. This fact is fundamentally related to the interplay between the different hypernucleus decay modes. A free $\Lambda$ particle decays essentially through the mesonic channel $\Lambda \rightarrow \mathscr{N}+\pi$. On the contrary when it is embedded in the nuclear medium the phase space for such a process is drastically reduced in all but the lightest hypernuclei $(\mathrm{A} \lesssim 10)$ due to the Pauli principle. At the same time the non-mesonic (or one-nucleon induced) decay mode $\Lambda+\mathscr{N} \rightarrow \mathscr{N}+\mathscr{N}$ opens up and becomes more and more dominant for increasing A. The $\tau$ measurement is then the doorway to determine both the hypernucleus mesonic and the non-mesonic decay widths as well as the ratio between the neutron to proton induced non-mesonic decay channels.

Several experiments provided $\tau$ values for light $\left({ }_{\Lambda}^{3} \mathrm{H},{ }_{\Lambda}^{4} \mathrm{H},{ }_{\Lambda}^{4} \mathrm{He},{ }_{\Lambda}^{5} \mathrm{He},{ }_{\Lambda}^{9} \mathrm{Be},{ }_{\Lambda}^{11} \mathrm{~B},{ }_{\Lambda}^{12} \mathrm{C}\right)$, medium $\left({ }_{\Lambda}^{27} \mathrm{Al},{ }_{\Lambda}^{28} \mathrm{Si},{ }_{\Lambda} \mathrm{Fe}\right)$ and heavy $\left({ }_{\Lambda}^{209} \mathrm{Bi},{ }_{\Lambda}^{238} \mathrm{U}\right)$ hypernuclei. The trend of the data shows a smooth decrease as a function of $\mathrm{A}$ and reaches a saturation value, corresponding to $80 \%$ of the free $\Lambda$ lifetime, above $\mathrm{A}=20$. From the experimental point of view it should be noted that such results were not obtained with the same methods. In fact they range from visualizing techniques to counter experiments. However for the heaviest systems it was impossible to measure the delay between their formation and their decay by applying direct timing methods, due to the overwhelming background of light particles. In these cases lifetime was inferred by applying the recoil shadow method [6] to the study of the fragments following delayed fission events induced by antiproton annihilation at rest. However these latter measurements were affected by large errors.

More recently the COSY-13 Collaboration performed new measurements of heavy mass hypernuclei [7], namely ${ }_{\Lambda}^{197} \mathrm{Au},{ }_{\Lambda}^{209} \mathrm{Bi}$ and ${ }_{\Lambda}^{238} \mathrm{U}$, at the Jülich COoler SYnchrotron. The leading idea of the experiment [8] was to exploit the high intensity of the COSY proton beam $\left(5 \times 10^{10}\right)$ in order to use very thin targets $\left(\leq 100 \mu \mathrm{m} / \mathrm{cm}^{2}\right)$ and to keep, at the same time, the event rate at a reasonable 

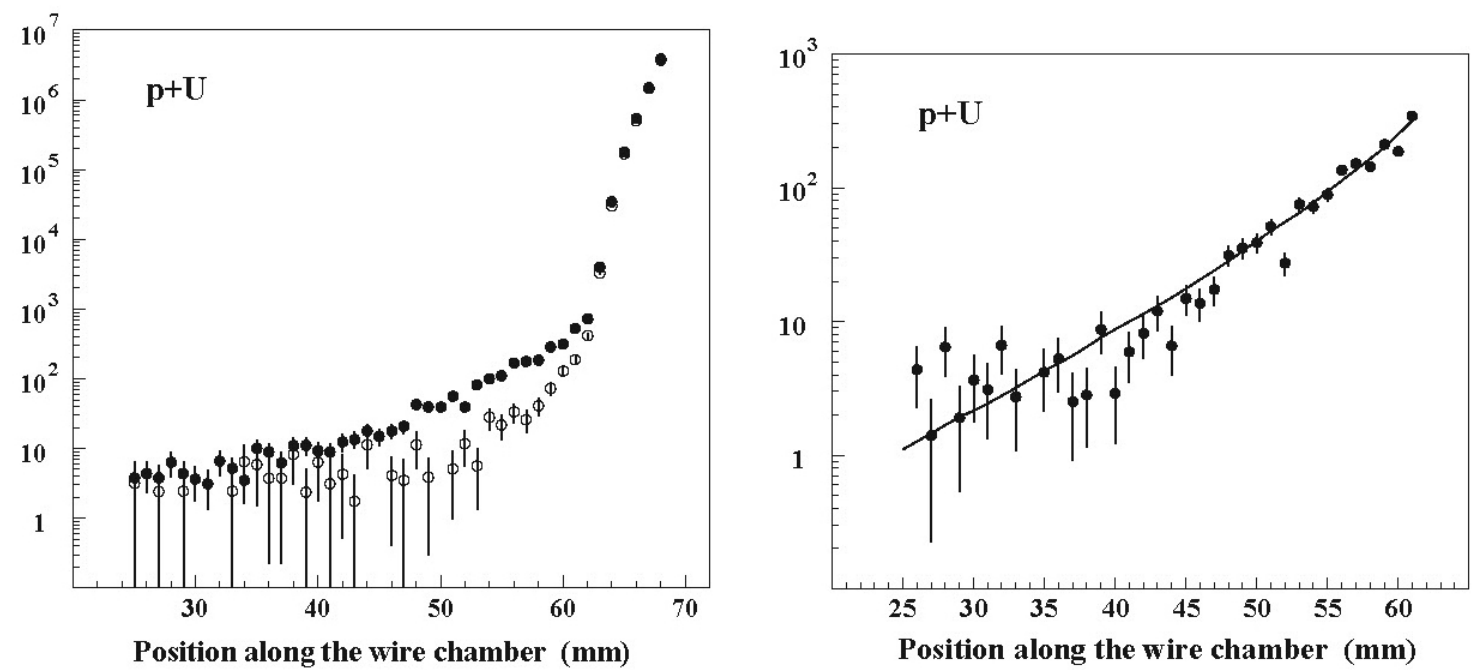

Figure 1: Left panel: spatial distribution of the measured hits of fission fragments following $p+U$ collisions. Full dots represent data for $T_{p}=1.9 \mathrm{GeV}$, whereas the open ones show data for $T_{p}=1.0 \mathrm{GeV}$, normalized to the bright part of the detectors during the $1.9 \mathrm{GeV}$ data taking. Right panel: spatial distribution of the measured hits of delayed fission fragments of hypernuclei in the shadow region, obtained by subtracting the background (normalized data taken at $1.0 \mathrm{GeV}$ ) from the spectrum measured at $1.9 \mathrm{GeV}$. Solid line shows the result of the simulation with the extracted value for the lifetime (from Ref. [7]e).

level. The reduced target thickness allowed to the hypernuclei produced in proton-nucleus collision to leave the target and to travel to some distance, proportional to the $\Lambda$ lifetime, before decaying. In this way it was possible to improve significantly the accuracy of the measurements. Fig. 1 shows the results obtained with the $U$ target. Similar distributions were observed for $A u$ and $B i$ targets. Table 1 summarizes all the COSY-13 results.

\begin{tabular}{|c|c|c|c|}
\hline Target & $\tau_{\Lambda}(\mathrm{ps})$ & $\tau_{\Lambda}(\mathrm{ps})$ & Ref. \\
\hline $\mathrm{Au}$ & $130 \pm 13_{\text {stat. }} \pm 15_{\text {syst. }}$ & $130 \pm 20$ & [7]c \\
\hline $\mathrm{Bi}$ & $161 \pm 7_{\text {stat. }} \pm 14_{\text {syst }}$. & $161 \pm 16$ & {$[7] \mathrm{b}$} \\
\hline $\mathrm{U}$ & $138 \pm 6_{\text {stat. }} \pm 17_{\text {syst }}$. & $138 \pm 18$ & [7]d \\
\hline
\end{tabular}

Table 1: Measured hypernuclear lifetimes (from Ref. [7]e). Errors reported in the third column were estimated by quadratically adding the statistical and the systematic ones.

These values, substantially in agreement with those obtained with antiprotons on same targets [9], led the COSY-13 Collaboration to claim that the phenomenological $\Delta I=\frac{1}{2}$ rule for $\Lambda+\mathscr{N} \rightarrow$ $\mathscr{N}+\mathscr{N}$ processes is violated, at a confidence level of 0.9 [7]c,e. However this hypothesis, independently suggested as well by Shumacher for light hypernuclei [10], was ruled out by the direct measurements of the ratio between the neutron to proton induced hypernucleus decay [11].

\subsection{The FINUDA experiment}

The FINUDA experiment is the paradigm of hypernuclear physics experiment successfully carried out where it was really hard even to imagine to do it, that is the INFN-LNF Double An- 
nular $\Phi$-factory for Nice Experiments (DA $\Phi N E$ ). The fundamental idea was to exploit the unique features of $K^{-}$following the $\phi$ resonance decay [12], namely low momentum, absence of contamination and $K^{+}$correlated emission. The indeed $K^{-}$low energy $(16.1 \mathrm{MeV})$ made possible to produce a large set of single $\Lambda$ hypernuclei by stopping them in very thin targets $(200 \div 300$ $\mathrm{mg} / \mathrm{cm}^{2}$ ). In this way the charged particles tagging both the formation and the decay of hypernuclei were measured with a very good resolution thanks to a dedicated spectrometer, designed in order to perform a complete program of hypernuclear studies [13]. The detector modeling phase was strongly influenced by the accelerator type and by the beam characteristics. The outcome was a typical collider apparatus with some important key features: very good overall performance in terms of detection efficiency and momentum resolution; large solid angle coverage and then capability of measuring in coincidence both the formation and the decay of the produced hypernucleus, possibility of using simultaneously up to 8 targets, made of different materials.

Recent results on non-mesonic hypernucleus weak decays, neutron-rich systems and low energy $K^{-}$interaction with nuclei are reported in Ref. [14] As far as hypernuclear spectroscopy is concerned, Fig. 2 shows the $\Lambda$ binding energy distribution measured for ${ }^{7} L i$ targets [15]. The picture shows clearly how difficult was to extract the result due to the large physical background.

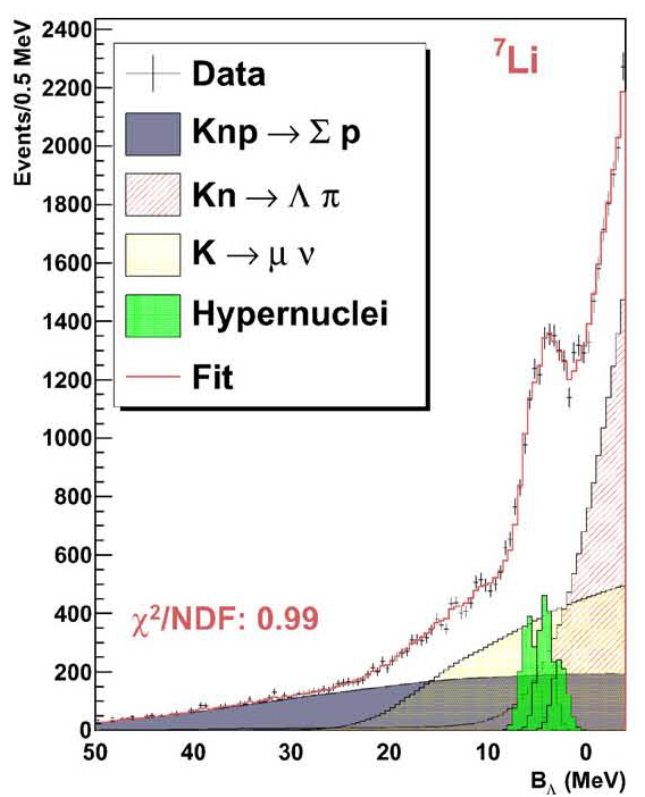

Figure 2: $\Lambda$ binding energy distribution measured by stopping $K^{-}$on ${ }^{7} \mathrm{Li}$ targets. The (colored) hatched areas depict the main background contributions (from Ref. [15]).

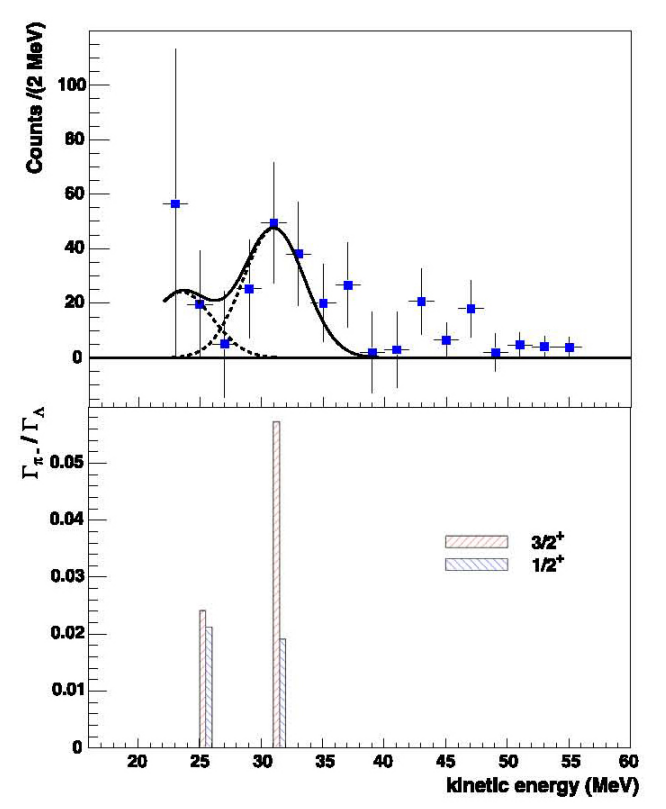

Figure 3: Upper panel: $\pi^{-}$kinetic energy distribution from ${ }_{\Lambda}^{15} \mathrm{~N}$ mesonic weak decay, after acceptance correction. The solid curve is a twogaussian fit to the peaks in the spectrum, to be compared with theoretical predictions reproduced in the lower panel; dashed curves are the single contributions. Lower panel: calculated major decay rates to final ${ }^{15} \mathrm{O}$ states: red bars outline the ${ }_{\Lambda}^{15} N$ ground state spin-parity $3 / 2^{+}$hypothesis, whereas blue ones correspond to the ${ }_{\Lambda}^{15} N$ ground state spin-parity $1 / 2^{+}$assumption (from Ref. [16]). 
However it is also evident that it was possible to keep it satisfactorily under control by identifying the main contributions to the continuum (listed in the histogram inset) and by well reproducing each of them. Similar results were obtained as well by stopping $K^{-}$on ${ }^{9} \mathrm{Be}[15],{ }^{12} \mathrm{C}[17],{ }^{13} \mathrm{C}[15]$ and ${ }^{16} \mathrm{O}[15]$ targets. For each observed hypernucleus it was possible to evaluate, for the first time, the formation probability. These series of data allowed for a systematic study of the hypernucleus properties as a function of A, in order to try to draw a comprehensive picture. It is worth to remind that such results, being obtained under identical experimental conditions, can be directly compared, essentially without need of taking into account possible systematic errors.

The set of spectroscopic information made available by the FINUDA experiment is completed by the systematic study of the hypernucleus mesonic weak decay charged channel. By exploiting at best the spectrometer capabilities, $\pi^{-}$spectra following the ${ }_{\Lambda}^{7} L i,{ }_{\Lambda}^{9} B e,{ }_{\Lambda}^{11} B$ and ${ }_{\Lambda}^{15} N$ mesonic weak decay were measured with magnetic analysis for the first time [16]. The shape of these distributions was interpreted through a comparison with pion distorted wave calculations that take into account the structure of both hypernucleus and daughter nucleus. Branching ratios $\Gamma_{\pi^{-}} / \Gamma_{\text {tot }}$ were derived from the measured spectra and converted to $\pi^{-}$decay rates $\Gamma_{\pi^{-}}$by means of known or extrapolated total decay widths $\Gamma_{\text {tot }}$. On the basis of these measurements the spin-parity assignment $1 / 2^{+}$for ${ }_{\Lambda}^{7} \mathrm{Li}$ and $5 / 2^{+}$for ${ }_{\Lambda}^{11} B$ ground state were confirmed whereas a spin-parity $3 / 2^{+}$for ${ }_{\Lambda}^{15} N$ was assigned for the first time (see Fig. 3).

\section{Future experimental initiatives}

\subsection{The PANDA experiment}

Some years ago the GSI management initiated a process to extend the existing facility and to offer to the scientific community an international laboratory where to perform research with antiprotons and ions (FAIR). The heart of the new accelerator complex is a double ring tunnel with circumference of 1100 meters, that will house 2 synchrotrons, SIS100 and SIS300. The synchrotron SIS100 will provide an intense $\left(4 \times 10^{13}\right)$ pulsed proton beam of $29 \mathrm{GeV}$ which is suitable for an efficient $\bar{p}$ production. Details about this project can be found in Ref. [18].

$\bar{p}$ will be finally available at the High Energy Storage Ring (HESR), a slow ramping synchrotron and storage ring equipped with stochastic and electron cooling to provide excellent beam energy definition. The energy could be varied from 3.0 up to $14.5 \mathrm{GeV}$.

The antiProton ANnihilation at DArmstadt Collaboration ( $\overline{\mathrm{P}} \mathrm{ANDA})$ is currently designing a multipurpose apparatus [19]. It will be installed on HESR to perform a large series of measurements with the aim of improving the knowledge of the strong interaction and of the hadron structure [20].

One of the main items of the physics program of this experiment is the study of the double $\Lambda$ hypernuclei. Despite the fact that the knowledge of $\Lambda \Lambda$ interaction represents a fundamental piece of information for reaching a unified understanding of the baryon-baryon interaction in the framework of SU(3) symmetry, experimental data on this subject are very scarce. Actually besides the first, pioneering measurements of ${ }_{\Lambda \Lambda}^{10} \mathrm{Be}$ [21] and ${ }_{\Lambda \Lambda}^{6} \mathrm{He}$ [22], only five different nuclear species with double strangeness were recently observed and unambiguously identified, namely ${ }_{\Lambda \Lambda}^{6} \mathrm{He},{ }_{\Lambda \Lambda}^{10} \mathrm{Be},{ }_{\Lambda \Lambda}^{11} \mathrm{Be},{ }_{\Lambda \Lambda}^{12} \mathrm{Be}$ and ${ }_{\Lambda \Lambda}^{13} B$ [23]. New, high statistics data samples are then highly desirable.

The most effective way to implant two units of strangeness in a nucleus is the $\Xi^{-}$atomic capture at rest. Usually $\Xi^{-}$production proceeds through the quasi-free elementary reaction 
$K^{-}+p \rightarrow \Xi^{-}+K^{+}$. An original and completely different experimental approach will be pursued at PANDA [24]. The basic idea is to produce a large number of $\bar{\Xi}^{+} \Xi^{-}$pairs trough $\bar{p}$-nucleus annihilations on a primary target. $\Xi^{-}$will be then slowed down in a secondary active target where it can form a double $\Lambda$ hypernucleus (or one or two hyperfragments). In addition the detection of $\bar{\Xi}^{+}$(or of $K^{+}$pair following its interaction in the primary target) will allow to tag the event.

This outlined program represents a very severe commitment since the study of double $\Lambda$ hypernuclei is neither just nor a simple extension of what has been done for the single $\Lambda$ hypernuclei. It must be reminded that, since $S=-2$ hypernuclei will be produced through a two-step mechanism, it will be no longer possible to perform spectroscopic study like in the case of $S=-1$ systems, that are generally formed in a two-body reaction. Identification and spectroscopic analysis of double $\Lambda$ hypernuclei can then only rely on the detection of their double sequential pionic decay. $\gamma$ spectroscopy could offer a possible way to partially overcome this problem. However this will represent a new challenge from the instrumental point of view. In fact $\bar{P}$ ANDA would be the first example of real, that is mechanical, integration of a magnetic spectrometer and a large array of HPGe detectors. To this purpose it has been experimentally demonstrated that the crystals can be safely operated immersed in a quite strong magnetic field [25].

\subsection{Supernuclear physics at $\operatorname{Super} B$ ?}

Following a long and successful tradition in building and operating lepton colliders, INFN recently launched a plan to construct the new $\operatorname{Super} B e^{+} e^{-}$complex accelerator [26]. SuperB is an INFN flagship project for a new high-luminosity heavy-flavor factory. Along with its companion detector [27], this new machine will be mainly dedicated to the measurements of $\mathrm{CP}$ violating processes in the $B$ meson sector, looking for deviations from the Standard Model predictions that can be interpreted as signals for new physics. At the same time the experiment will perform highprecision tests of the Standard Model. Super $B$ will be an asymmetric, double-ring accelerator designed to run at the $\Upsilon(4 S)$ resonance center-mass (c.m.) energy with a baseline luminosity in excess of $10^{36} \mathrm{~cm}^{-2} \mathrm{~s}^{-1}$, that is about two orders of magnitude larger than the peak luminosity of the existing $B$-factories. The nominal beam energies will be $\mathrm{E}_{e^{+}} \approx 6700 \mathrm{MeV}$ and $\mathrm{E}_{e^{-}} \approx 4200$ $\mathrm{MeV}$ but Super $B$ will have the capability of running in the c.m. energy range between $\psi(3770)$ (the charm threshold) and $\Upsilon(5 S)$ resonance as well. A more detailed description of this challenging INFN commitment can be found in Ref. [28], while the complete, wide spectrum of SuperB physics reach is described in Ref. [29].

However SuperB could offer as well the opportunity for a systematic, high-statistics study of $b$ baryon properties and for a search for bottom and, possibly, charmed supernuclei, that is bound nuclear systems with an explicit content of beauty or charm. The possible existence of such objects, where for instance a $\Lambda_{c}^{+}$replaces a constituent nucleon of a nucleus, was suggested several years ago by Tyapkin [30], in complete analogy to what happens with $s$ baryon $\Lambda$ which can replace one nucleon in a nucleus to form nuclear bound systems. This idea is a very natural and straightforward extension grounded on the expectation that the entire family of baryons experiences exchange forces which are similar to the corresponding interaction potentials. This statement is strengthened by the close similarity of the quark structure of the lightest flavored baryons $\Lambda$ (uds), $\Lambda_{c}^{+}(\mathrm{udc})$ and $\Lambda_{b}^{0}(\mathrm{udb})$. Despite the large difference between $\Lambda$ and $\Lambda_{c}^{+}$lifetime and mass values, theoretical calculations based on such assumed universality of the $\Lambda_{i}-\mathscr{N}$ interaction $(i=s, c$, 
$b, t, \ldots)$ actually lead to predict a rich spectrum of charmed supernuclei, spanning over a quite wide range of atomic numbers [31-36]. However they provide very different estimates about the binding energy and the potential-well depth. By keeping in mind that $\Lambda_{b}^{0}$ is obviously not sensitive to the Coulomb force, the situation for bottom supernuclei should be even more promising.

More recently this argument triggered a renewed interest, at least from the theoretical point of view [37, 38]. In the latter works, $\Lambda_{c}^{+}$and $\Lambda_{b}^{0}$ supernuclei were systematically and quantitatively studied in the frame of the quark-meson coupling (QMC) model [39]. Although results are encouraging, experimental evidences of the existence of either charmed or bottom supernuclei are still missing, with the exception of three ambiguous candidates of $\Lambda_{c}^{+}$supernucleus observed in a series of emulsion experiments $[40,41]$. This unsatisfactory scenario either could lead to the conclusion that the basic hypothesis is wrong or could be ascribed to the limitations of the beams available and of the experimental techniques exploited so far [42].

In this context Super $B$ could offer the opportunity to make a real highly desired breakthrough in this intriguing subject. The main idea, described in details in Ref. [43], consists in installing a thin target around the interaction point in order to intercept as many $B^{-}$following the $\Upsilon(4 S)$ resonance decay, in order to produce $b$ baryons $\left(\Lambda_{b}^{0}, \Sigma_{b}^{ \pm}, \Sigma_{b}^{* \pm}\right.$ and, possibly, $\left.\Xi_{b}^{-0}\right)$ via the beauty exchange reaction induced on nuclei. It is indeed worth to remind that this experimental approach, although experimentally very hard, is the only possible way to infer information about $b$ baryonnucleon interaction at low energies.

\section{References}

[1] M. Danysz and J. Pniewsky, Philos. Mag. 44 (1953) 348.

[2] O. Hashimoto, H. Tamura, Prog. Part. Nucl. Phys. 57 (2006) 564.

[3] W. M. Alberico, G. Garbarino, Phys. Rep. 369 (2002) 1 [nucl-th/0112036].

[4] Y. Akaishi, T. Yamazaki, Phys. Rev. C65 (2002) 044005; T. Yamazaki, Y. Akaishi, Phys. Lett. B535 (2002) 70.

[5] L. Majling, Nucl. Phys. A585 (1995) 211c.

[6] V. Metag et al. Nucl. Instr. Meth. 114 (1974) 445.

[7] H. Ohm et al., Phys. Rev. C55 (1997) 3062;

P. Kulessa et al., Phys. Lett. B427 (1998) 403;

B. Kamys et al., Eur. Phys. J. A11 (2001) 1 [hep-ex/0011063];

P. Kulessa et al., Acta Phys. Polon. B33 (2002) 603 [nucl-ex/0108027];

W. Cassing et al., Eur. Phys. J. A16 (2003) 549 [nucl-ex/0109012].

[8] K. Pysz et al., Nucl. Instrum. Meth. A420 (1999) 356.

[9] T. A. Armstrong et al., Phys. Rev. C47 (1993) 1957.

[10] R. A. Schumacher, Nucl. Phys. A547 (1992) 143C.

[11] M. Kim et al., Phys. Rev. Lett. 103 (2009) 182502.

[12] T. Bressani, in proceedings of Workshop on Physics and Detector for DAФNE, Frascati (RM), Italy, April 9-12, 1991, p. 475.

[13] A. Feliciello, Riv. Nuovo Cim. 32 (2009) 147. 
[14] S. Bufalino et al., this volume; E. Botta et al., this volume; A. Filippi et al., this volume.

[15] M. Agnello et al. [FINUDA Collaboration], Phys. Lett. B698 (2011) 219 [nucl-ex/1011.2695].

[16] M. Agnello et al. [FINUDA Collaboration], Phys. Lett. B681 (2009) 139 [nucl-ex/0 905 . 0623 ].

[17] M. Agnello et al. [FINUDA Collaboration], Phys. Lett. B622 (2005) 35 [nucl-ex/0506028].

[18] A. Lehrach, this volume.

[19] $\bar{P} A N D A$ Collaboration, Strong Interaction Studies with Antiprotons, FAIR-ESAC/Pbar/Technical Progress Report (2005) 1.

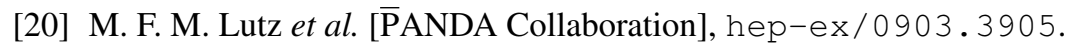

[21] M. Danysz et al., Nucl. Phys. 49 (1963) 121.

[22] D. J. Prowse, Phys. Rev. Lett. 17 (1966) 782.

[23] K. Nakazawa [KEK-E176 and J-PARC-E07 Collaborations], Nucl. Phys. A835 (2010) 207.

[24] J. Pochodzalla, Nucl. Instrum. Meth. B214 (2004) 149.

[25] T. Bressani and A. Feliciello, Nucl. Phys. News. 20 (02) (2010) 23 and references therein.

[26] M. E. Biagini et al. [SuperB Collaboration], physics . acc-ph/1009.6178.

[27] E. Grauges et al. [SuperB Collaboration], physics.ins-det/1007.4241.

[28] F. Wilson, this volume.

[29] D. G. Hitlin et al., hep-ph/0810.1312.

[30] A. A. Tyapkin, Yad. Fiz. 22 (1975) 181.

[31] S. Iwao, Lett. Nuovo Cim. 19 (1977) 647.

[32] C. B. Dover and S. H. Kahana, Phys. Rev. Lett. 39 (1977) 1506.

[33] R. Gatto and F. Paccanoni, Nuovo Cim. A46 (1978) 313.

[34] N. N. Kolesnikov et al., Sov. J. Nucl. Phys. 34 (1981) 533 [Yad. Fiz. 34 (1981) 957].

[35] G. Bhamathi, Phys. Rev. C24 (1981) 1816;

G. Bhamathi, Nuovo Cim. A102 (1989) 607.

[36] H. Bandō and M. Bando, Phys. Lett. B109 (1982) 164.

[37] C. H. Cai, L. Li, Y. H. Tan, P. Z. Ning, Europhys. Lett. 64 (2003) 448.

[38] K. Tsushima and F. C. Khanna, Prog. Theor. Phys. Suppl. 149 (2003) 160; [nucl-th/0212100]; K. Tsushima and F. C. Khanna, Phys. Lett. B552 (2003) 138 [nucl-th/ 207036 ]; K. Tsushima and F. C. Khanna, Phys. Rev. C67 (2003) 015211 [nucl-th/0207077]; K. Tsushima and F. C. Khanna, J. Phys. G30 (2004) 1765 [nucl-th/0303073].

[39] P. A. M. Guichon, Phys. Lett. B200 (1988) 235.

[40] Yu. A. Batusov et al., Pisma Zh. Eksp. Teor. Fiz. 33 (1981) 56.

[41] V. V. Lyukov, Nuovo Cim. A102 (1989) 583.

[42] N. I. Starkov and V. A. Tsarev, in proceedings of 1986 INS International Symposium on Hypernuclear Physics, Tokyo, Japan, August 10-23, 1986, p. 246;

N. I. Starkov and V. A. Tsarev, Nucl. Phys. A450 (1986) 507c.

[43] A. Feliciello, T. Bressani and V. Lucherini, in proceedings of Hadron 2011 - XIV International Conference on Hadron Spectroscopy, Munich, Germany, June 13-17, 2011, eConf C110613 (2011); T. Bressani, A. Feliciello, V. Lucherini, this volume. 